\title{
Space-time analysis of geopotential height and SLP, intraseasonal oscillations, weather regimes, and local climates over the North Atlantic and Europe
}

\author{
Eric Simonnet ${ }^{1, *}$, Guy Plaut ${ }^{2}$ \\ ${ }^{1}$ Laboratoire de Météorologie dynamique du CNRS, Ecole Polytechnique, 91128 Palaiseau, France \\ ${ }^{2}$ Institut Non-Linéaire de Nice CNRS, Université de Nice-Sophia Antipolis, 1361 route de Lucioles, 06560 Valbonne, France
}

\begin{abstract}
We extract coherent and robust oscillations at the intraseasonal time scale using multichannel singular spectrum analysis (MSSA). Three daily fields are compared, namely sea-level pressure (SLP) and geopotential heights at 500 and $700 \mathrm{hPa}$ (Z500 and Z700). The data set extends from 1958 to 1997 and covers the same North Atlantic-European domain. Three oscillations stand out with periods of 30-35, 65-70 and 120-130 d respectively. A comparison shows that the same period oscillations at different levels are highly correlated. Moreover, it is shown that these modes are phaselocked and thus should come out from a single limit cycle. When these oscillations are compared with the succession of weather regimes (WRs), it can be shown that WR occurrences are indeed strongly influenced by particular phases of the 30-35 and 65-70 d modes. Transition from one WR to another is also shown to be favored by the life cycle of the previous modes. For instance, zonal regimes are followed preferably by blocking ones. Thus, regime transitions are indeed not random but are somehow steered by the dynamical behavior of a low-dimensional atmospheric attractor. We then explore the links between the intraseasonal modes and the local temperatures over France and Western Europe as well as precipitations over France. Once again, we conclude that bursts of low-frequency oscillations, such as the ones detected, significantly influence local surface weather conditions.
\end{abstract}

KEY WORDS: Multichannel singular spectrum analysis (MSSA) - Intraseasonal oscillations · Sealevel pressure (SLP) $\cdot$ Geopotential height $\cdot$ Weather regimes

\section{INTRODUCTION}

Dynamical system theory provides a challenging description of the atmosphere. The existence of a strange attractor of finite dimension with complex topological structure is now recognized likely to be at the core of the nonlinear dynamics of the atmosphere. Spells of regular activities such as quasi-stationary behavior (unstable fixed points) and regular oscillations (unstable limit cycles) are characteristic of this attractor. Low-frequency oscillations (LFOs) related to unstable limit cycles have become a major subject of interest; they cover a large frequency range from intermonthly, interannual up to interdecadal periods. Multichannel singular spectrum analysis (MSSA) enables one to extract these unstable limit cycles; it is a

*E-mail: esimon@lmd.ens.fr powerful adaptive tool which allows the identification of intermittent and robust oscillations. It has been extensively presented in many papers and the reader is referred to Plaut \& Vautard (1994) for more details.

On the other hand, we could also view the atmosphere as a (discrete) set of points in a phase space of large but finite dimension. These points correspond in this case to (anomaly) maps such as daily large-scale circulation (LSC) sea-level pressure (SLP), Z700 and $Z 500$ patterns. Some regions of the phase space are more visited than others and correspond to maxima of the corresponding probability density function (PDF). In order to detect these highly populated regions, the notion of clusters has been introduced: a cluster centroid corresponds more or less to an accumulation point in the phase space. The partition of the phase space into different clusters thus gives an idea of the most visited regions, which are called for convenience 
weather regimes (WRs). The dynamical cluster algorithm (Michelangeli et al. 1995) provides an objective way to split the phase space into different clusters together with a measure of their statistical stability. In this case, the cluster centroids correspond to accumulation points (the algorithm minimizes the sum of variances within clusters), that is, to recurrent atmospheric states.

Since the motion within the phase space is primarily governed by the dynamics of the attractor, it is tempting to explore if LSC regimes and particular phase of oscillations are connected. In Plaut \& Vautard (1994), the quasi-stationary LSC regimes of Vautard (1990) were compared with LFOs, on a 32 yr set of National Meteorological Center (NMC) analysis for the geopotential heights at $700 \mathrm{hPa}(Z 700)$. In this work, the clusters regimes are identified by another method (the dynamical cluster algorithm), which favors recurrence instead of quasi-stationary behavior. The duration of a given WR does not exceed $10 \mathrm{~d}$ on average, so we focus especially on intraseasonal LFOs.

In Section 2, we systematically extract intraseasonal LFOs from 3 fields (Z700, Z500 and SLP) of analysed or observed data from different sources: the National Centers for Environmental Prediction (NCEP) and the Climate Research Unit (CRU). The results are then compared at the 3 different levels. In Section 3 we try to understand the connections between these oscillations and the LSC WRs. Section 4 focuses on the connection between LFOs and local temperatures and precipitation, and Section 5 is the conclusion.

\section{COMPARISONS BETWEEN Z700, Z500 AND SLP LOW-FREQUENCY OSCILLATIONS}

\subsection{Data and methodology}

The daily $Z 500$ and $Z 700$ data we use come from the NCEP/NCAR reanalysis project and extend from 1958 to 1997. The SLP data set covers a longer period of $120 \mathrm{yr}(1880-1997)$ and corresponds to instantaneous data measurements collected at the CRU (see Jones 1987). To make comparison easier, we use the same $100^{\circ}$ longitude, $40^{\circ}$ latitude wide window as used in Michelangeli et al. (1995) and Plaut \& Simonnet (2001 this issue) $\left(-60\right.$ to $40^{\circ} \mathrm{W}, 30$ to $\left.70^{\circ} \mathrm{N}\right)$. We choose a $10^{\circ}$ longitude $\times 5^{\circ}$ latitude grid for SLP and a $5^{\circ}$ longitude $\times 5^{\circ}$ latitude grid for $Z 500$ and $Z 700$. The data are centered by subtracting the seasonal trend (that is, the trend is calculated in a seasonal way, i.e. is calendartime dependent) and are sampled every other day. The resulting data are then spatially prefiltered by a classical principal component analysis (PCA): the original set is projected onto the leading empirical orthogonal functions (EOFs), which give the directions of maximal variance in phase space. A few leading eigenmodes are retained, and the corresponding spatial principal components (PCs) form the channels to be analysed by MSSA. We always took the leading 6 PCs as the number of channels; the first 6 EOFs accounted for $75 \%$ of the total variance in the 3 cases. Identical results were obtained when selecting the first 10 EOFs (85 to $90 \%$ of the total variance).

The core idea of MSSA is to decompose a multichannel series: $\vec{X}(t+s)=\sum a_{k}(t) \vec{E}_{k}(s)$. The discrete analogue is thus to consider $X_{1, i}$ as

$$
X_{1, i+j}=\sum_{k=1}^{L \times M} a_{i}^{k} E_{l j}^{k}, 1 \leq 1 \leq L, 1 \leq j \leq M
$$

1 is the index corresponding to the channels (for instance, the PCs obtained through PCA) and $i$ is a time discretisation. $E_{l j}^{k}$ correspond to orthonormal adaptive space-time structures called ST-EOFs. They are the eigenvectors of a multi-lag $(M)$ multi-channel $(L)$ cross-covariance matrix. The size of the problem is $L \times M$ ST-EOFs, where $L$ is the number of channels and $M$ is the time window length used, that is, each STEOF can be considered as a temporal sequence of an $L$-dimensional spatial structure of duration $M$. The computation then results in a set of ST-EOFs associated with their projection coefficients $a_{i}^{k}$ called ST-PCs. PCA and SSA are particular cases of MSSA with $M=1$ and $L=1$ respectively. A nice feature of MSSA is its ability to extract oscillatory behavior provided intermittent but recurrent oscillatory space-time patterns are present within the data set. The LFOs manifest themselves through the existence of pairs of oscillatory ST-EOFs with similar period and in-phase quadrature; the corresponding cross-covariance matrix eigenvalues are (nearly) degenerated. We use the same criterion as in Plaut \& Vautard (1994) to select the oscillatory pairs corresponding to LFOs. In order to study the space-time structure of an oscillatory pair, we perform a composite analysis keyed to the phase of the oscillation. That is, we basically split the life cycle of the oscillation of period $T$ into 8 equally populated sets of length $T / 8$. This is done by performing a new PCA on the reconstructed oscillation (a precise definition of the reconstructed algorithm is given in Plaut \& Vautard 1994) and then using the phase space direction which has the maximal reconstructed variance, that is, the first PC obtained. The instantaneous phase $\varphi$ of the oscillation is defined as the polar angle in the plane of this PC and its time-derivative. 8 phase sectors (or categories) are defined according to the value of $j$, for instance, category 1 days are days for which $-22.5^{\circ}<\varphi$ $<22.5^{\circ}$. Phase composites keyed to the oscillation are then computed by averaging the involved field for all category 1 days, then category 2 days, etc. 
In the following, we perform at least 3 MSSA with respective window lengths of 75, 150 and $300 \mathrm{~d}$ and adopt the notation SLP_ $M$ for a particular MSSA of the field SLP for instance, with a window length $M$ days.

\subsection{SLP}

The results of the different MSSA experiments are shown in Table 1. The spatial and spectral behavior of the LFOs are described. Pairs of eigenvalues which do not exactly meet the criteria for oscillatory pairs but which are nevertheless physically significant are shown in italics. A general feature is that LFOs are particularly robust (both for their periods and their space-time patterns) with regard to changing MSSA parameters; sensitivity experiments are described in Section 2.2.2.

The $31 \mathrm{~d}$ oscillation (SLP_75,150,300) is characteristic of a westward propagation of a dipole pattern along $60^{\circ} \mathrm{N}$. The phase composites of the SLP anomalies are shown in Fig. 1. The ST-EOFs 10 and 11 together account for only $2.7 \%$ of the total variance (annual cycle removed). This is only an average; such an oscillation is essentially intermittent, with periods of inactivity which are responsible for the relatively small total variance accounted for. However, the ratio between the power spectrum of the corresponding STPCs and the total power spectrum of the multichannel time series giving the local (in time) variance accounted for may reach more than $15 \%$ at the maximum of activity. We thus conclude that such an oscilla-

Table 1. Characteristics of the oscillatory pairs identified. The first column contains the name of the experiment, the second column the order of the pair. The third column corresponds to the period of the oscillation calculated as the peak value of the sum of the SSA-ME spectra of the 2 PCs. In the fourth column the total percentage of variance accounted for is reported (first $6 \mathrm{PCs}$ ). The last column gives the physical characteristic of the oscillation. S-N: northward propagation; E-W: westward propagation. Italics: cases where pairs of eigenvalues are not exactly in phase quadrature, i.e. criteria for oscillatory pairs have not been met

\begin{tabular}{|lcccc|}
\hline Expt & Pair & Period (d) & Variance (\%) & Type \\
\hline SLP_150 & $\mathbf{4 - 5}$ & $\mathbf{6 6}$ & $\mathbf{4 . 1}$ & S-N \\
& $\mathbf{1 0 - 1 1}$ & $\mathbf{3 1}$ & $\mathbf{2 . 7}$ & E-W \\
& $2-3$ & 121 & 4.7 & $S-N$ \\
& $13-14$ & 41 & 2.5 & $E-W$ \\
SLP_75 & $\mathbf{6 - 7}$ & $\mathbf{3 2}$ & $\mathbf{4 . 5}$ & $\mathbf{E - W}$ \\
& $24-25$ & 27 & 2.3 & $E-W$ \\
& $27-28$ & 15 & 2.0 & E-W \\
SLP_300 & $\mathbf{2 - 3}$ & $\mathbf{1 3 3}$ & $\mathbf{2 . 6}$ & S-N \\
& $\mathbf{8 - 9}$ & $\mathbf{6 9}$ & $\mathbf{2 . 2}$ & $\mathbf{S - N}$ \\
& $\mathbf{2 1 - 2 2}$ & $\mathbf{3 0}$ & $\mathbf{1 . 4}$ & $\mathbf{E - W}$ \\
& & & & \\
\hline
\end{tabular}

tion is significant. We also give the (spectral) variance of the oscillation accounted for at the peak of frequency, which reaches $26 \%$ in this case (see also Plaut \& Vautard 1994).

The $65 \mathrm{~d}$ oscillation corresponds to a northward propagation of the anomalies (SLP_150, pair 4-5); phase composites are shown in Fig. 2. A $121 \mathrm{~d}$ oscillation (pair 2-3, SLP_150, 300) is also present and has the same spatial structure as the $65 \mathrm{~d}$ mode, with northward propagation of the anomalies. There is possibly a $40 \mathrm{~d}$ mode associated with the pair 13-14 (SLP_150) but which is not robust enough. We also found variability around 27 and $15 \mathrm{~d}$ (pairs 24-25 and 27-28). They correspond to a westward propagation.

We next compare the 3 oscillations, that is, the 120 , 65 and $31 \mathrm{~d}$ oscillations. We study the correspondence between the 8 phases of a given oscillation and the 8 phases of another oscillation. It is represented by an $8 \times 8$ contingency table. Significance bounds are computed with a random Monte Carlo procedure: we randomly shuffle the 8 phases' indices 100 times, then compute the 100 corresponding $8 \times 8$ contingency tables, and sort the 100 percentages we get for each member of these contingency tables. Finally we compare the observed percentages to the 5th and 95th sorted percentages. This procedure is also described in Plaut \& Vautard (1994). The 31 d oscillation is intermittently phase-locked with the $65 \mathrm{~d}$ mode: the histogram of simultaneous occurrences of the 2 oscillations shows 2 preferred categories of the $31 \mathrm{~d}$ mode with one $65 \mathrm{~d}$ mode phase category (Table 2 and composites of Figs 1

Table 2. Contingency table between the 8 phase indices of the 31, 65 and 133 d SLP oscillations. Bold: above the $95 \%$ significance level; italics: below the $5 \%$ level

\begin{tabular}{|c|c|c|c|c|c|c|c|c|}
\hline & 1 & 2 & 3 & 4 & 5 & 6 & 7 & 8 \\
\hline \multicolumn{9}{|c|}{31 d/65 d (5-95 \%) } \\
\hline 1 & 235 & 258 & 168 & 212 & 234 & 275 & 190 & 225 \\
\hline 2 & 207 & 275 & 226 & 181 & 204 & 263 & 230 & 198 \\
\hline 3 & 193 & 246 & 290 & 148 & 186 & 233 & 266 & 212 \\
\hline 4 & 212 & 210 & 279 & 200 & 195 & 204 & 257 & 216 \\
\hline 5 & 211 & 192 & 226 & 293 & 181 & 194 & 259 & 240 \\
\hline 6 & 188 & 187 & 192 & 281 & 214 & 218 & 227 & 265 \\
\hline 7 & 234 & 191 & 203 & 252 & 283 & 198 & 216 & 245 \\
\hline 8 & 264 & 190 & 191 & 209 & 268 & 241 & 214 & 214 \\
\hline \multicolumn{9}{|c|}{$65 d / 133 d(5-95 \%)$} \\
\hline 1 & 233 & 270 & 227 & 142 & 231 & 282 & 208 & 151 \\
\hline 2 & 176 & 302 & 238 & 175 & 185 & 236 & 274 & 163 \\
\hline 3 & 156 & 255 & 305 & 229 & 141 & 209 & 271 & 209 \\
\hline 4 & 186 & 180 & 328 & 245 & 191 & 169 & 258 & 219 \\
\hline 5 & 230 & 128 & 229 & 295 & 241 & 143 & 224 & 275 \\
\hline 6 & 231 & 200 & 150 & 244 & 275 & 213 & 222 & 291 \\
\hline 7 & 273 & 244 & 160 & 212 & 288 & 240 & 168 & 274 \\
\hline 8 & 322 & 206 & 174 & 227 & 254 & 261 & 199 & 172 \\
\hline
\end{tabular}


1
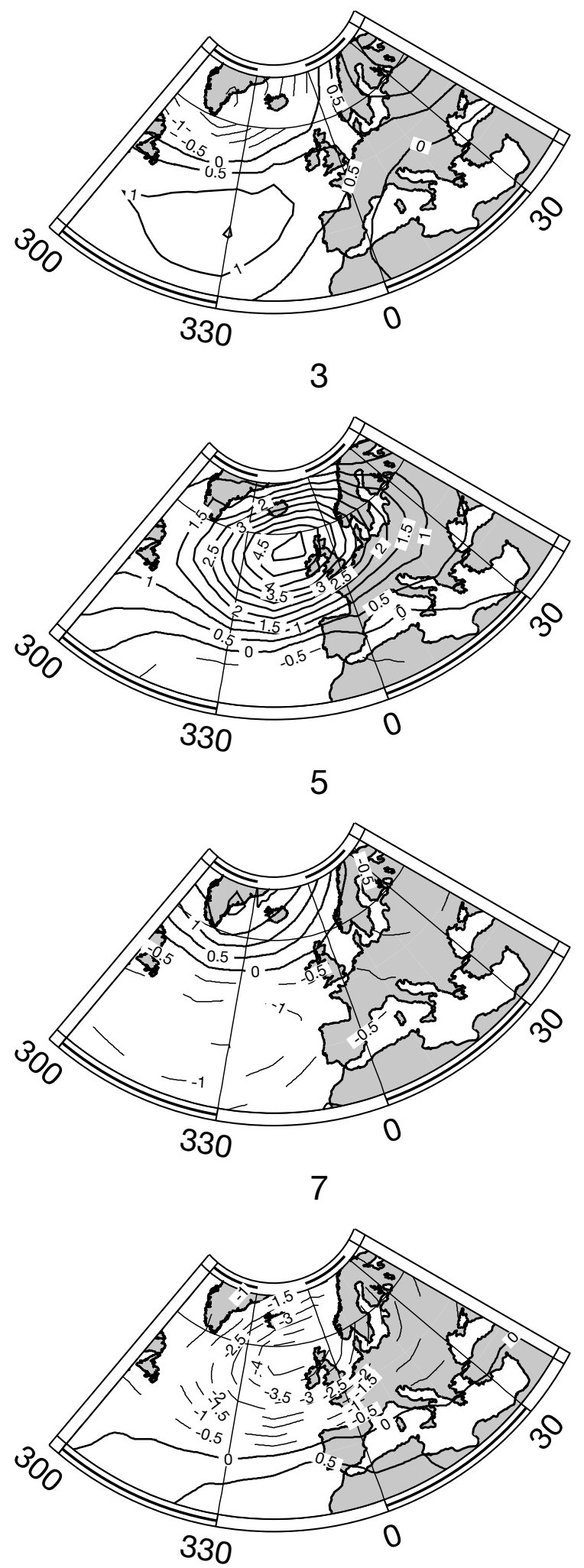

2
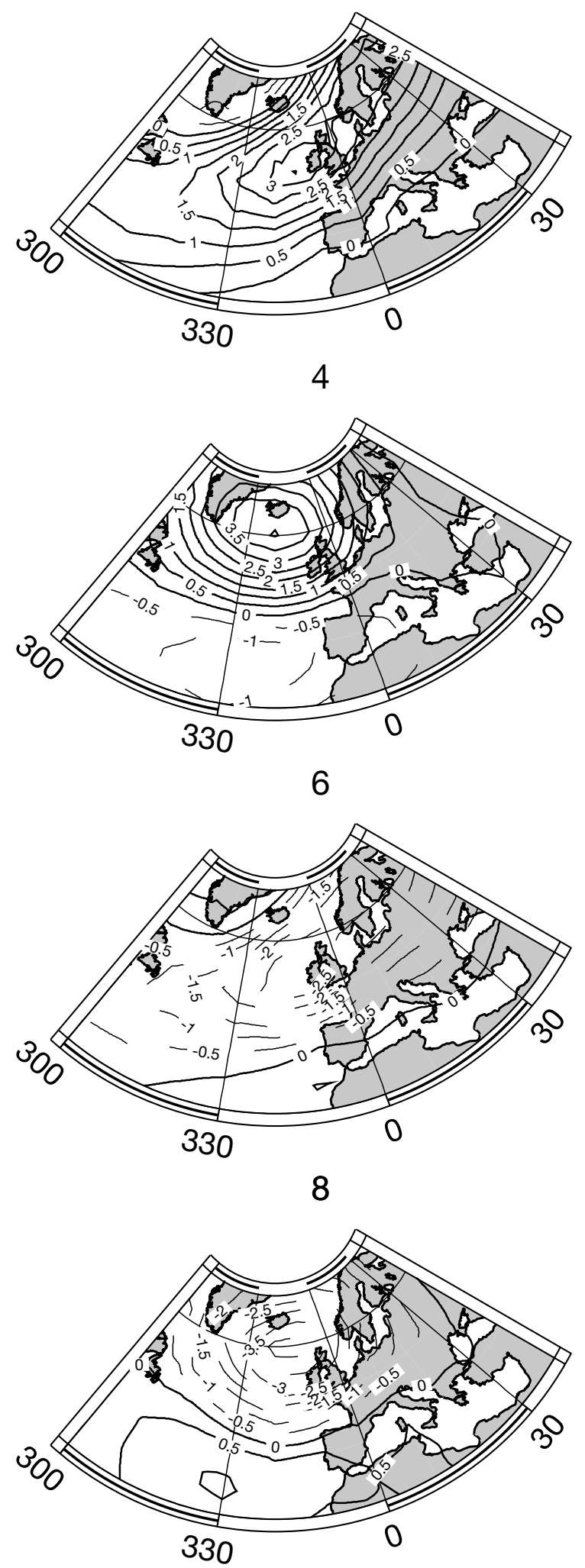

Fig. 1. Composite of the SLP anomalies keyed to phase categories 1 to 8 of the $31 \mathrm{~d}$ oscillation (pair 10-11). Contour interval: $0.5 \mathrm{hPa}$ 

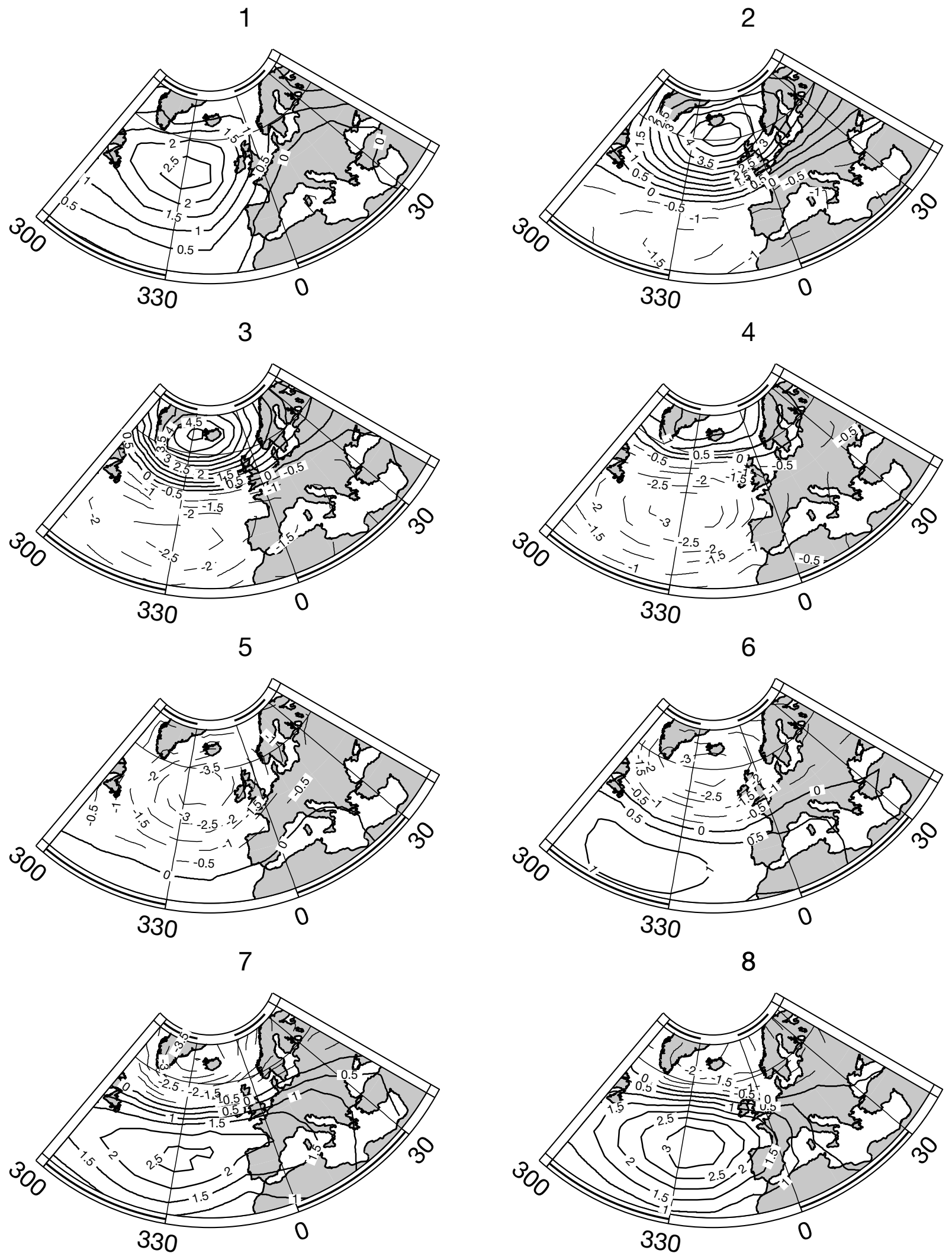

Fig. 2. Composite of the SLP anomalies keyed to phase categories 1 to 8 of the 65 d oscillation (pair 4-5, SLP_150). Contour interval: $0.5 \mathrm{hPa}$ 
\& 2). This is reminiscent of what was observed in Plaut \& Vautard (1994) for the $Z 700$ field and for their 30-35 and $70 \mathrm{~d}$ oscillations, which exhibited the same spatiotemporal structures as our limit cycles and a similar phase-locking. The nonlinear interaction between the 2 modes is apparent in Fig. 2, where phase 6 has an anomaly much less pronounced than in the opposite phase 2. Phase 6 of the $65 \mathrm{~d}$ mode is locked with phases 1 and 2 of the $31 \mathrm{~d}$ mode with opposite anomalies (see Table 2). A close inspection reveals that phases 7 and 1 have less-pronounced anomalies than the opposite phases 3 and 5 .

We also observe a more pronounced (intermittent) phase-locking between the 125-130 d oscillation and the $65 \mathrm{~d}$ mode. The 31 and $65 \mathrm{~d}$ modes are likely to be harmonics of the 125-130 d oscillation, meaning that these 3 modes preferably belong to a unique limit cycle in the phase space.

\subsubsection{LFOs further in the past}

It is tempting to ask if the LFOs above were also present before 1958. We thus consider the same experiments but using 2 different historical periods. The first one corresponds to period from 1880 to 1918, the second from 1918 to 1958. In the first case, we obtain the 2 phase-locked LFOs but with different periods of around $25 \mathrm{~d}$ for the retropropagating dipole and around $50 \mathrm{~d}$ for the other. The $133 \mathrm{~d}$ mode is present but now with a smaller period of $100 \mathrm{~d}$. The second data set also shows similar patterns: we have at least 3 distinct and robust modes which are similar to the $60 \mathrm{~d}$ mode and propagate northward, but with different periods of roughly 40,60 and $80 \mathrm{~d}$. It is worthy to note that the corresponding ST-EOFs are in this case exactly the same but with different periods and probably represent the same physical oscillation with its frequency modulated. Associated with these oscillations is a $20 \mathrm{~d}$ mode with a westward propagation, probably related to what is known as the Branstator oscillation. Both indeed correspond to westward propagating Rossby waves. The last experiment consists of performing MSSA for the $120 \mathrm{yr}$ long period and detecting intraseasonal oscillations similar to the previous ones. A somewhat disappointing result is that oscillatory pairs disappear in this case (no pairs in phase quadrature). The ST-EOFs detected are indeed spatially similar to the previous ones. This reinforces the conclusion that shift in the frequencies of the 3 locked oscillations is significant and is characteristic of the very-low-frequency (interdecadal or lower) behavior of these oscillations. As a matter of fact, MSSA has to deal with a (rather large) continuum of frequencies instead of a mere monochromatic (even noisy) signal and is thus unable to give 1 pair of ST-EOFs. It is the reason why MSSA gives ST-EOFs with similar spatial structures but different periods. ST-EOFs have actually to be dilated or contracted in some way to fit different historical periodic oscillation behavior. A coupling between MSSA and wavelet analysis is likely to resolve this kind of problem. We save this for future studies.

\subsubsection{Sensitivity experiments}

It has been shown recently than SSA is able to detect spurious oscillations (Allen \& Smith 1996). However, Monte Carlo tests are far from simple to implement in the context of MSSA. In order to test robustness to parameter changes, we prefer to follow the same approach as in Plaut \& Vautard (1994): we conduct the 2 following experiments. We first divide the data into 2 sets of approximately $20 \mathrm{yr}$ and perform MSSA. We recover the 3 oscillations in the 2 cases with the same periods and space-time structures. We then split the data (spanning the period 1958-1997) into 1 set holding the first $5 \mathrm{yr}$ of any decade and the other set consisting of the last $5 \mathrm{yr}$ (that is, the years ending with 5, $6,7,8$ or 9). Once again MSSA detects the same 3 oscillations (Table 3). Similar tests with geopotentials also confirm the robustness of the oscillations found. Thus, we may actually stress the relevance of our results. Indeed, to check the significance of any pair of eigenvalues, the authors of Allen \& Smith (1996) generated thousands of surrogate time series. On page 3382 of Allen \& Smith (1996), they stress that 'any finite segment of red noise will contain more power than the process average at some frequencies due simply to statistical fluctuations'. As in Plaut \& Vautard (1994), we divided the actual time series into non-overlapping subsets in several ways. If the oscillations were originating from random power enhancement at some frequencies, we would not find the pairs at the same frequencies and with the same propagative scheme using non-overlapping time series. We also checked that the

Table 3. Sensitivity experiment. Characteristics of the oscillatory pairs for SLP: years ending with $0,1,2,3$ or 4 (SLPa) and years ending with $5,6,7,8$ or 9 (SLPb)

\begin{tabular}{|lcccc|}
\hline Expt & Pair & Period (d) & Variance (\%) & Type \\
\hline SLPa_300 & $3-4$ & 125 & 2.5 & S-N \\
& $8-9$ & 67 & 2.0 & S-N \\
& $11-12$ & 42 & 1.8 & E-W \\
& $22-23$ & 31 & 1.5 & E-W \\
SLPb_300 & $4-5$ & 67 & 2.5 & S-N \\
& $11-12$ & 42 & 1.8 & E-W \\
& $21-22$ & 31 & 1.4 & E-W \\
\hline
\end{tabular}


existence of our oscillations was very weakly dependent on the precise definition of the Euro-Atlantic domain. Let us add that Plaut \& Vautard (1994) already observed sectorial oscillations like ours.

\subsection{Geopotentials}

The MSSA results are summarized in Table 4 for $Z 700$ and Table 5 for $Z 500$.

\subsection{1. $Z 700$}

We observe at least 4 oscillations. The $31 \mathrm{~d}$ mode appears together with a (Branstator) $21 \mathrm{~d}$ mode. Both oscillations correspond to a westward retropropagation of a dipole pattern. Their corresponding ST-EOFs have similar structures. The existences of 65 and 120-130 d LFOs are confirmed when choosing a larger window length of $300 \mathrm{~d}$. In this case the 31-65-133 d limit cycle appears again very clearly. We compute the contingency tables between these modes and the 3 corresponding SLP modes: the 3 LFOs are perfectly in phase (contingency numbers highly significant), showing that this complex oscillation is also present in the

Table 4. Characteristics of the oscillatory pairs for Z700. C: clockwise rotation; $\mathrm{AC}$ : anticlockwise rotation

\begin{tabular}{|lcccc|}
\hline \multicolumn{1}{l}{ Expt } & Pair & Period (d) & Variance (\%) & Type \\
\hline Z700_150 & $11-12-13$ & 31 & 3.9 & E-W \\
& $22-23-24$ & 21 & 3.1 & E-W \\
& $2-3$ & 120 & 4.3 & S-N \\
Z700_300 & $4-5$ & 66 & 3.7 & S-N \\
& $2-3$ & 133 & 2.4 & S-N \\
& $7-8$ & 66 & 2.0 & S-N \\
& $12-13$ & 43 & 1.8 & C \\
& $23-24$ & 30 & 1.4 & E-W \\
& $25-26$ & 76 & 1.4 & AC \\
\hline
\end{tabular}

Table 5. Characteristics of the oscillatory pairs for $Z 500$. E-SW: southwest propagation

\begin{tabular}{|lrccl|}
\hline \multicolumn{1}{l}{ Expt } & Pair & Period (d) & Variance (\%) & Type \\
\hline Z500_150 & $12-13$ & 31 & 2.5 & E-W \\
& $23-24$ & 23 & 2.0 & E-W \\
& $8-9$ & 43 & 3.0 & C \\
Z500_300 & $2-3$ & 142 & 2.3 & S-N \\
& $7-8$ & 66 & 1.9 & S-N \\
& $10-11-12$ & 43 & 2.6 & E-W \\
& $16-17$ & 50 & 1.5 & E-SW \\
& $20-21$ & 33 & 1.4 & E-W \\
& $25-26$ & 29 & 1.3 & E-W \\
\hline
\end{tabular}

Table 6. Contingency table between the 8 phase indices of the 65 d SLP and 65 d Z700 oscillations. Bold: statistical significance $(\mathrm{p}<0.05)$

\begin{tabular}{|rrrrrrrrr|}
\hline & 1 & 2 & 3 & 4 & 5 & 6 & 7 & 8 \\
\hline 1 & $\mathbf{7 8 1}$ & 122 & 60 & 33 & 21 & 14 & 87 & $\mathbf{5 9 9}$ \\
2 & $\mathbf{5 6 5}$ & $\mathbf{8 0 3}$ & 148 & 37 & 20 & 30 & 41 & 113 \\
3 & 119 & $\mathbf{5 3 8}$ & $\mathbf{8 3 5}$ & 141 & 59 & 50 & 33 & 43 \\
4 & 40 & 78 & $\mathbf{5 2 8}$ & $\mathbf{8 2 8}$ & 160 & 56 & 46 & 35 \\
5 & 35 & 38 & 80 & $\mathbf{5 6 8}$ & $\mathbf{7 6 1}$ & 153 & 76 & 15 \\
6 & 25 & 43 & 32 & 92 & $\mathbf{6 4 3}$ & $\mathbf{7 9 5}$ & 139 & 84 \\
7 & 115 & 50 & 43 & 39 & 71 & $\mathbf{6 5 6}$ & $\mathbf{8 1 7}$ & 111 \\
8 & 98 & 77 & 49 & 38 & 30 & 71 & $\mathbf{6 2 0}$ & $\mathbf{8 1 5}$ \\
& & & & & & & & \\
\hline
\end{tabular}

atmosphere with the same triple phase-locking. The phase composites of the 3 Z700 modes are exactly similar to the 3 SLP modes and are not shown here. Table 6 gives the correspondence between the $65 \mathrm{~d}$ SLP and $65 \mathrm{~d} Z 700$ oscillations. Two oscillations with periods of 43 and $76 \mathrm{~d}$ are also present. The first one is indeed perfectly in phase with the weak $41 \mathrm{~d}$ SLP mode found before but is less sensitive to window length: it is robust and its space-time structure is different than the other modes. It corresponds more or less to a clockwise rotation with a south-westward trajectory of the anomaly over Europe followed by a westward propagation over the southern Atlantic. The second, $76 \mathrm{~d}$ oscillation is phase-locked with the $65 \mathrm{~d}$ mode, although it corresponds to an anticlockwise rotation of a dipole centered roughly west of the British Isles but is sensitive to changes of window length.

\subsection{2. $Z 500$}

We recover once again the same 31-65-130 d limit cycle as for $Z 700$ and SLP. The composites are very similar to the SLP LFOs. The histogram of simultaneous occurrences of both phase categories shows that these oscillations are identical in both cases. We also find the same 23 and $43 \mathrm{~d}$ modes of $Z 700$ (they are perfectly in phase; the contingency tables are not shown here). The 29 d mode (Z500_300 experiment) is, in fact, phase-locked with the $33 \mathrm{~d}$ mode, and its pattern is very similar (westward retropagation along $60^{\circ} \mathrm{N}$ ). We also obtain a $50 \mathrm{~d}$ oscillation with a different spatial structure propagating south-westward, which seems to be present for the $Z 500$ field only.

\subsection{3. $Z 500-Z 700$}

In order to detect possible baroclinic oscillations, we performed MSSA on the difference between the 2 geopotential fields. We slightly increased the number 
of EOFs to 10 to capture enough variance. One significant and very robust oscillation only appears, with period of $42 \mathrm{~d}$. It is thus strongly suspected to be related to the previous $Z 500$ and $Z 70042 \mathrm{~d}$ modes. Although contingency tables between them are not strictly diagonal, they show a net phase-locking between the different phases of the 3 oscillations. Phase composites of the $Z 500$ and $Z 700$ fields according to the phase of the $Z 500$ minus $Z 70042 \mathrm{~d}$ oscillating pair are then performed: both are similar but reveal that the $Z 700$ gradient is less pronounced than the $Z 500$ gradient during the life cycle of the oscillation. We thus have in this case an oscillation which is indeed merely a trace of the $42 \mathrm{~d}$ geopotential mode due to gradient differences. We thus conclude that, apparently, no genuine baroclinic oscillations exist at this time scale. Note that similar conclusions were drawn in the study of the difference between the $Z 700$ and Z300 fields.

\section{LOW-FREQUENCY OSCILLATIONS AND WEATHER REGIMES}

In the companion paper (Plaut \& Simonnet 2001) 5 SLP WRs, representative of the extended winters (from November to March), are selected by the dynamical cluster algorithm for the period 1958-1997, namely Blocking (BL, or EA for European Anticyclone), Western Blocking (WBL), Atlantic Ridge (AR), Zonal (ZO) and Greenland Anticyclone (GA). The classification of all-year LSCs gives the same 5 clusters (after removing the seasonal cycle and trend and keeping the first 6 spatial PCs). The clusters obtained for $Z 500$ and $Z 700$ keeping daily patterns through the year are identical at both levels and more robust than when a classification using only winters is made (see Plaut \& Simonnet 2001); they are the already well-known BL and $\mathrm{ZO}$ and a third one called AR-GA (it corresponds to a mixing of AR and GA). The differences in the classification between the different levels result from the fact that one projects a density probability function describing the state of the whole atmosphere (all levels included) into 3 different directions in the phase space (that is, SLP and geopotential phase spaces). It naturally results in slight differences between the projected clusters obtained at the 3 levels (which are strongly related to maxima of the densities), as well as different statistical significances.

As observed in Plaut \& Vautard (1994), particular phases of these oscillations are indeed very similar to some of the LSC clusters found using the dynamical cluster algorithm described above. For instance, the 65 d mode (Fig. 2) is perfectly described with the 4 clus- ters AR, WBL, GA and ZO: AR corresponds to a positive anomaly starting in the south of the Atlantic. If this anomaly is shifted northward, it becomes a WBL anomaly followed by a GA pattern and finally a ZO-like anomaly corresponding to the end of the half-cycle (more or less the opposite of WBL). This suggests that there exists a dynamical link between these LSC weather regimes. As one could remark, the LFOs described above take only a small percentage of the total variance and are certainly not the whole explanation for the transitions between WRs. These transitions are often determined by transient phenomena and synoptic activities, but such phenomena could also contribute to the LFO cycle.

In order to study the connections between these oscillations and WRs, we consider the previous classifications of all-year LSCs and the reconstructions of the two 31-65 d modes for the 3 different fields (using a $300 \mathrm{~d}$ window length). We then analyse the links between oscillations and WRs.

\subsection{SLP}

3.1.1. Description of the reconstructed 31 and $65 \mathrm{~d}$ oscillations in terms of WRs

Given the 31 and 65 d reconstructed oscillations, it is possible to describe them in terms of our 5 WRs. For each day, we look for the best pattern correlation between the isolated reconstructed oscillation and the cluster centroids; this defines the (fictitious) WR occurring on this day for the reconstruction alone. We then count the number of occurrences of transitions of each type (including persistence) between such defined WR at Day $n$ and WR at Day $n+1$. We thus obtain a precise description of the 2 oscillations in terms of these fictitious LSC regimes. The results are given in Table 7 . It is largely consistent with a visual comparison of the different phases of the LFO and the WR patterns. The $31 \mathrm{~d}$ mode is characterized by the transitions $\mathrm{ZO} \rightarrow \mathrm{BL} \rightarrow \mathrm{WBL} \rightarrow \mathrm{GA} \rightarrow \mathrm{ZO}$. The $\mathrm{AR}$ regime, roughly centered in the south of the Atlantic domain, is poorly represented, since the anomaly propagates along $60^{\circ} \mathrm{N}$ and westward. It can also be seen that the LFO spends much more time in the WBL and ZO states, which corresponds precisely to opposite phases of the limit cycle. The $65 \mathrm{~d}$ mode is symmetric to the $31 \mathrm{~d}$ mode in terms of the AR and $\mathrm{BL}$ clusters, that is, the BL cluster, centered on the eastern Europe, is badly represented, since the anomaly propagates across the Atlantic. Once again the LFO spends more time in the WBL and ZO states but also the GA state. The transitions between fictitious WRs are $\mathrm{ZO} \rightarrow \mathrm{AR} \rightarrow \mathrm{WBL} \rightarrow \mathrm{GA} \rightarrow \mathrm{ZO}$. 
Table 7. Number of transitions between fictitious regimes at Day $n$ (columns) and fictitious regimes at Day $n+1$ (lines) for the 31 and $65 \mathrm{~d}$ reconstructed SLP oscillations. Bold: preferred transitions

\begin{tabular}{|lrrrrr|}
\hline & BL & ZO & GA & AR & WBL \\
\hline 31 d & & & & & \\
BL & 1677 & 4 & 3 & 7 & $\mathbf{4 3 3}$ \\
ZO & $\mathbf{4 2 4}$ & 4052 & 4 & 53 & 0 \\
GA & 5 & $\mathbf{4 6 4}$ & 2164 & 0 & 4 \\
AR & 12 & 12 & 11 & 280 & 46 \\
WBL & 6 & 0 & $\mathbf{4 5 5}$ & 21 & 4171 \\
$\mathbf{6 5} \mathbf{d}$ & & & & & \\
BL & 350 & 4 & 1 & 17 & 11 \\
ZO & 23 & 3581 & 1 & $\mathbf{1 7 4}$ & 0 \\
GA & 9 & $\mathbf{1 9 4}$ & 3841 & 0 & 3 \\
AR & 1 & 1 & 2 & 2363 & $\mathbf{1 8 7}$ \\
WBL & 0 & 0 & $\mathbf{2 0 2}$ & 0 & 3343 \\
\hline
\end{tabular}

\subsubsection{Link between reconstructed oscillation and observed WRs}

We now count for each of the 8 phases of the 2 oscillations (in this case pairs $4-5$ and 10-11, SLP_150) the number of actual occurrences of the 5 WRs. Note that for each oscillatory pairs the time behavior of the category number turns out to be very regular, with successive values of $1,2,3, \ldots, 8,1, \ldots$ We thus obtain the contingency table shown in Table 8. It confirms the fact that the actually preferred transitions regimes occurring in the atmosphere are $\mathrm{ZO} \rightarrow \mathrm{BL}(\mathrm{AR}) \rightarrow \mathrm{WBL} \rightarrow$

Table 8. Number of days corresponding to the 5 observed regimes (BL, ZO, GA, AR, WBL) and simultaneously belonging to a given phase category of the 31 and $65 \mathrm{~d}$ SLP oscillations. Bold: above the $95 \%$ significance level; italics: below the $5 \%$ level

\begin{tabular}{|lccccc|}
\hline & BL & ZO & GA & AR & WBL \\
\hline $\mathbf{3 1} \mathbf{d}$ & & & & & \\
1 & $\mathbf{4 9 6}$ & 388 & 237 & 344 & 332 \\
2 & $\mathbf{5 0 6}$ & 257 & 197 & 359 & $\mathbf{4 6 5}$ \\
3 & 362 & 199 & 229 & $\mathbf{4 0 5}$ & $\mathbf{5 7 9}$ \\
4 & 285 & 239 & 366 & 334 & $\mathbf{5 4 9}$ \\
5 & 370 & 278 & $\mathbf{4 9 4}$ & 314 & 340 \\
6 & 302 & $\mathbf{4 0 9}$ & $\mathbf{5 0 6}$ & 327 & 228 \\
7 & 337 & $\mathbf{5 6 8}$ & $\mathbf{4 2 1}$ & 319 & 177 \\
8 & 364 & $\mathbf{5 7 8}$ & 333 & 319 & 197 \\
$\mathbf{6 5} \mathbf{d}$ & & & & & \\
1 & 326 & 312 & 234 & $\mathbf{3 8 8}$ & $\mathbf{4 8 4}$ \\
2 & 351 & 201 & 352 & 271 & $\mathbf{5 7 4}$ \\
3 & 315 & 196 & $\mathbf{5 5 8}$ & 218 & $\mathbf{4 8 8}$ \\
4 & 338 & 276 & $\mathbf{5 9 4}$ & 263 & 305 \\
5 & 381 & $\mathbf{4 4 5}$ & $\mathbf{4 2 6}$ & 284 & 229 \\
6 & $\mathbf{4 2 4}$ & $\mathbf{5 5 7}$ & 283 & $\mathbf{3 3 8}$ & 224 \\
7 & $\mathbf{4 7 3}$ & $\mathbf{4 9 0}$ & 179 & $\mathbf{4 5 5}$ & 262 \\
8 & $\mathbf{4 1 4}$ & $\mathbf{4 3 9}$ & 157 & $\mathbf{5 0 4}$ & 301 \\
\hline
\end{tabular}

$\mathrm{GA} \rightarrow \mathrm{ZO}$, which is to be expected from Table 7. This was also observed in Plaut \& Vautard (1994) for the Z700 field, but Vautard (1990) defined regimes by requiring that the average over all occurrences of neighboring patterns of the instantaneous tendencies vanishes, instead of minimizing the sum of intra-cluster variance (dynamical cluster algorithm).

We then compare the observed transitions between WRs, with the previous transitions and the fictitious transition of Section 3.1.1. We count the number of transitions between the 5 SLP WRs; the corresponding contingency table is given in Table 9. The Monte Carlo procedure described in Section 2.2 is slightly modified so that persistency within a given regime is not taken into account. We obtain a remarkable consistency between the observed preferred transitions of Table 9 and the LFOs transitions of Tables $7 \& 8$. The 2 noticeable exceptions are the transitions $\mathrm{ZO} \rightarrow \mathrm{GA}$ and $\mathrm{WBL} \rightarrow \mathrm{AR}$, which do not seem to follow the life cycle of the 31 and $65 \mathrm{~d}$ SLP oscillations. The transition GA $\rightarrow$ BL could be explain by the life cycle of the $65 \mathrm{~d}$ oscillation (Table 8).

Table 9. Number of transitions between regimes occurring at Day $n$ (columns) and regimes at Day $n+1$ (lines). Bold: above the $95 \%$ significance level; italics: below the $5 \%$ level

\begin{tabular}{|lccccr|}
\hline & BL & ZO & GA & AR & WBL \\
\hline BL & - & $\mathbf{2 8 0}$ & 142 & 235 & $\mathbf{2 6 5}$ \\
ZO & $\mathbf{2 0 8}$ & - & $\mathbf{1 7 4}$ & $\mathbf{1 9 8}$ & 1 \\
GA & $\mathbf{2 6 4}$ & 125 & - & 90 & 148 \\
AR & 226 & 175 & 152 & - & $\mathbf{2 1 7}$ \\
WBL & 225 & 1 & $\mathbf{1 5 8}$ & $\mathbf{2 4 7}$ & - \\
\hline
\end{tabular}

\section{2. $Z 700$ and $Z 500$}

We consider the same experiments as before and consider the 3 clusters AR-GA, ZO and BL. The 31-65 d oscillations are the same as for SLP; it is thus not surprising that we obtain the transitions $\mathrm{ZO} \rightarrow$ $\mathrm{BL} \rightarrow \mathrm{AR}-\mathrm{GA} \rightarrow \mathrm{ZO}$ for the $31 \mathrm{~d}$ oscillation as well as for the $65 \mathrm{~d}$ mode. Table 10 displays the number of days of each WR falling into the phase categories of the 3 main oscillations for the $Z 700$ field. It indicates clearly the preference for the transitions $\mathrm{ZO} \rightarrow \mathrm{BL} \rightarrow$ AR-GA $\rightarrow$ ZO. This is in agreement with the corresponding phase composites.

\section{LOW-FREQUENCY OSCILLATIONS AND SURFACE DATA}

We are now interested in the relation between the main intraseasonal oscillations identified before, that 
Table 10. Number of days corresponding to the observed regimes $\mathrm{ZO}, \mathrm{BL}$ and $\mathrm{AR}-\mathrm{GA}$ and simultaneously belonging to a given phase category of the 31 and $42 \mathrm{~d} Z 700$ oscillations. Bold: above the $95 \%$ significance level; italics: below the $5 \%$ level

\begin{tabular}{|llll|}
\hline $\mathbf{3 1} \mathbf{d}$ & ZO & BL & AR-GA \\
\hline 1 & $\mathbf{6 8 8}$ & $\mathbf{7 0 8}$ & 461 \\
2 & 564 & $\mathbf{7 9 5}$ & 475 \\
3 & 533 & $\mathbf{7 5 8}$ & 548 \\
4 & 507 & 639 & $\mathbf{7 1 9}$ \\
5 & 575 & 569 & $\mathbf{7 1 0}$ \\
6 & $\mathbf{7 2 9}$ & 485 & $\mathbf{6 4 1}$ \\
7 & $\mathbf{8 0 0}$ & 474 & 559 \\
8 & $\mathbf{8 1 5}$ & 529 & 509 \\
$\mathbf{6 5} \mathbf{d}$ & & & \\
1 & 602 & $\mathbf{6 3 1}$ & 543 \\
2 & 449 & $\mathbf{6 7 4}$ & $\mathbf{6 9 4}$ \\
3 & 481 & 564 & $\mathbf{8 3 5}$ \\
4 & 547 & 622 & $\mathbf{6 6 1}$ \\
5 & $\mathbf{6 6 9}$ & 585 & 533 \\
6 & $\mathbf{8 5 3}$ & 577 & 480 \\
7 & $\mathbf{8 5 6}$ & $\mathbf{6 7 3}$ & 379 \\
8 & $\mathbf{7 5 4}$ & 631 & 497 \\
$\mathbf{4 2} \mathbf{d}$ & & & \\
1 & $\mathbf{6 6 8}$ & 661 & 559 \\
2 & 591 & $\mathbf{7 1 9}$ & 530 \\
3 & 492 & $\mathbf{7 5 6}$ & 567 \\
4 & 529 & $\mathbf{7 0 9}$ & $\mathbf{6 3 7}$ \\
5 & 587 & 581 & $\mathbf{6 8 2}$ \\
6 & $\mathbf{7 2 9}$ & 487 & $\mathbf{6 3 7}$ \\
7 & $\mathbf{8 5 1}$ & 472 & 508 \\
8 & $\mathbf{7 6 4}$ & 572 & 502 \\
\hline & & & \\
\hline & & & \\
\hline
\end{tabular}

is, the 31 and $65 \mathrm{~d}$ modes, and the local weather, namely, temperatures for France and for Europe. We also compare these oscillations with precipitations (Météo-France data set).

\subsection{Data and methodology}

Our first surface weather data set contains temperatures from 30 Météo-France stations. Daily precipitation heights from the same 30 stations are also available. The other data set contains NCEP reanalysed temperatures over Europe (702 grid points). They both cover the period 1958-1997.

The life cycle of the 31 and $65 \mathrm{~d}$ modes is first divided into 8 equally populated sets (pairs $4-5$ and 10-11, SLP_150). This composite analysis provides a daily succession of the phase indices of the 2 oscillations over the period 1958-1997. We then process the Météo-France station data as for the daily mean temperatures for the same period; for instance, we classify these temperatures into 3 equally populated classes: the cold, mean and warm terciles. We finally compare the succession of these 3 indices with the succession of the 8 phases of the limit cycles obtained using MSSA, and we compute the corresponding $3 \times 8$ contingency tables, with the significance bounds computed by the Monte Carlo procedure described in Section 2.2. For instance, we count the number of days with simultaneous occurrences of a warm temperature at the selected location and phase $n$ of a reconstructed LFO. We compute the percentage of warm days for phase $n$ which we compare to $33 \%$, the climatic percentage of warm days (by definition of terciles). If the percentage is, for instance, $40 \%$, we conclude that it changed by a factor of $(40-33) / 33=21 \%$. (the pairs $8-9$ and $21-22$ of SLP_300 give the same results, showing that the result is fairly independent of the MSSA parameters). Note that it is exactly the same approach as in Plaut \& Simonnet (2001), where they make a comparison between the occurrence of regimes and the behavior of local data over a given area. The only difference is that the regimes are now replaced by the 8 phase indices of an LFO.

\subsection{Temperatures}

The results are shown in Fig. 3 for the $31 \mathrm{~d}$ mode and Fig. 4 for the $65 \mathrm{~d}$ mode. They represent fields of percentage deviations from the expected $33 \%$ together with the shaded area representing nonsignificant deviations (inside the 5-95\% confidence level interval). During phase 3 (and also phase 2) of the $31 \mathrm{~d}$ oscillation, we observe a net tendency to have more cold days than average. The opposite phase 7 (but also phase 8) clearly shows the reverse tendency to have more warm days than average. This is consistent with the previous results that link phases 2 and 3 preferably to WBL regime patterns and phases 7 and 8 to zonal regimes (see composites in Fig. 2). One must be aware that this oscillation does obviously interact with temperature tendencies, although its contribution to the total variance in the atmosphere is weak. The $65 \mathrm{~d}$ mode seems to interact more than the $31 \mathrm{~d}$ mode with the temperatures: the deviation tendencies are more pronounced during the cold phases 1, 2 and 3 and warm phases 5,6 and 7. It is also in agreement with the corresponding weather regimes and Fig. 2 (see also Tables 7 to 9). For instance, phase 6 corresponds to anomalous zonal flows, which explains why it is a warm phase over most of Europe. The fact that it is phase-locked with cold phases 1 and 2 of the $31 \mathrm{~d}$ mode and is nevertheless a warm phase should not be discounted: in the ideal case where the 2 modes would be perfectly phase-locked, the number of days in phase 6 would account for only $50 \%$ of the total days in phases 1 and 2 of the $31 \mathrm{~d}$ mode. It is precisely this dif- 


\section{SLP 31-day LFO (warm days)}

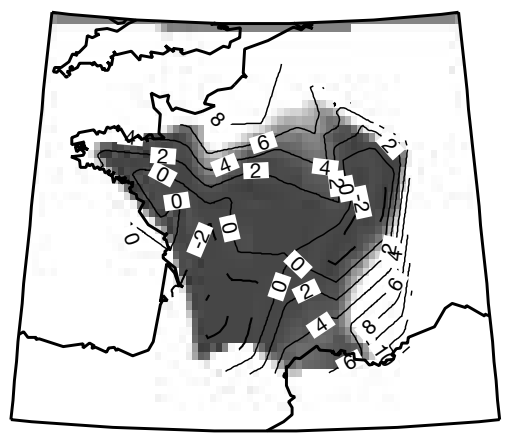

Phase 1

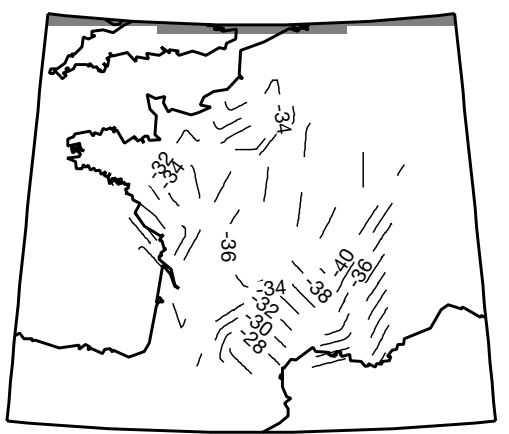

Phase 3

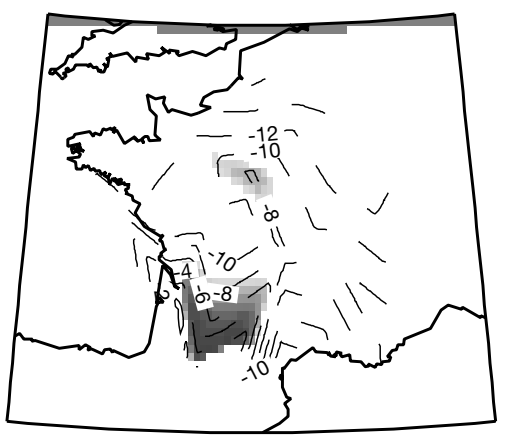

Phase 5

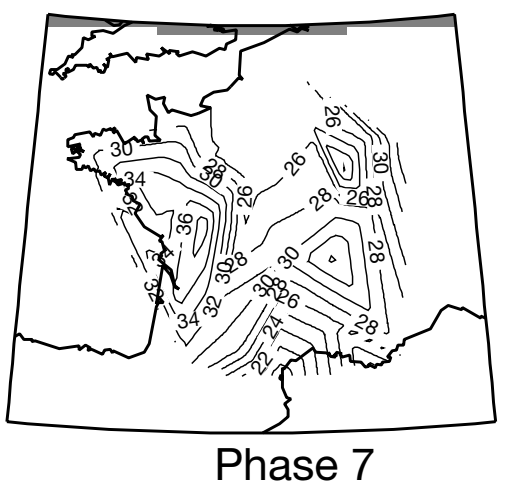

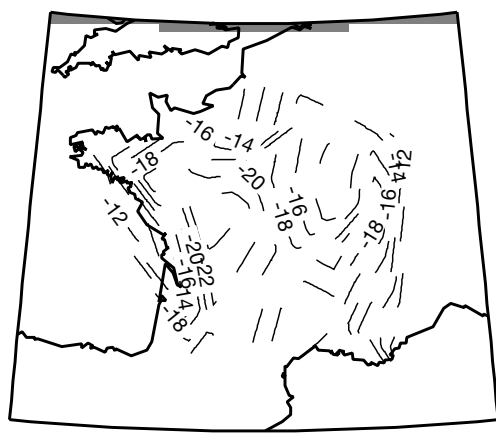

Phase 2

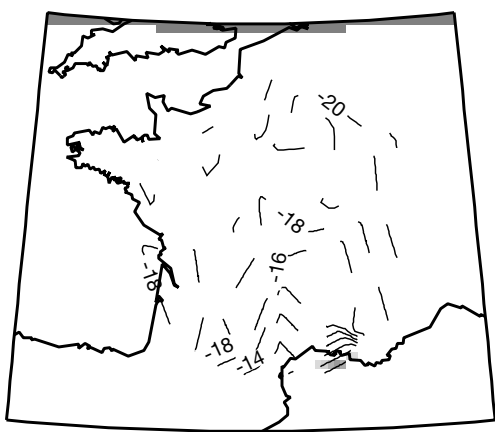

Phase 4

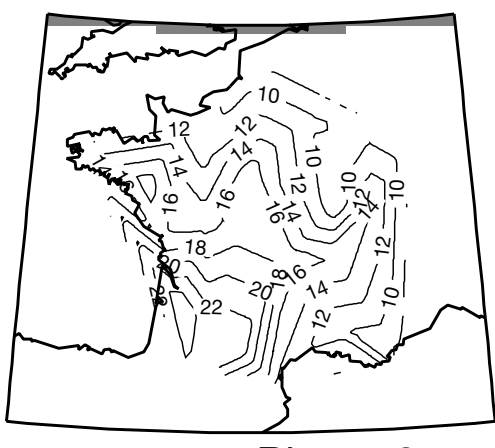

Phase 6

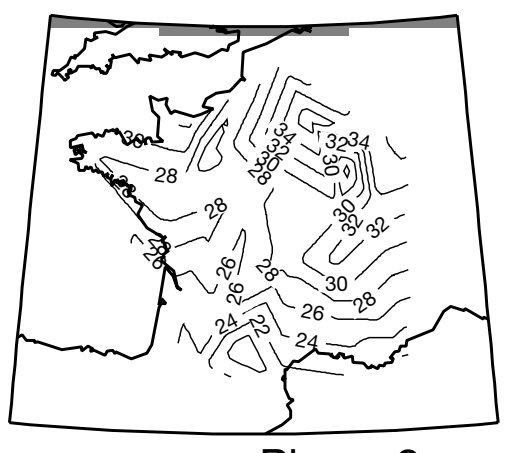

Phase 8

Fig. 3. Relation between the 8 phases of the $31 \mathrm{~d}$ oscillation and warm days (third tercile): contour levels (percentage deviation from the expected $33 \%$ ). The shaded area corresponds to regions where the deviations are between the 5 and $95 \%$ confidence levels 


\section{SLP 65-day LFO (warm days)}
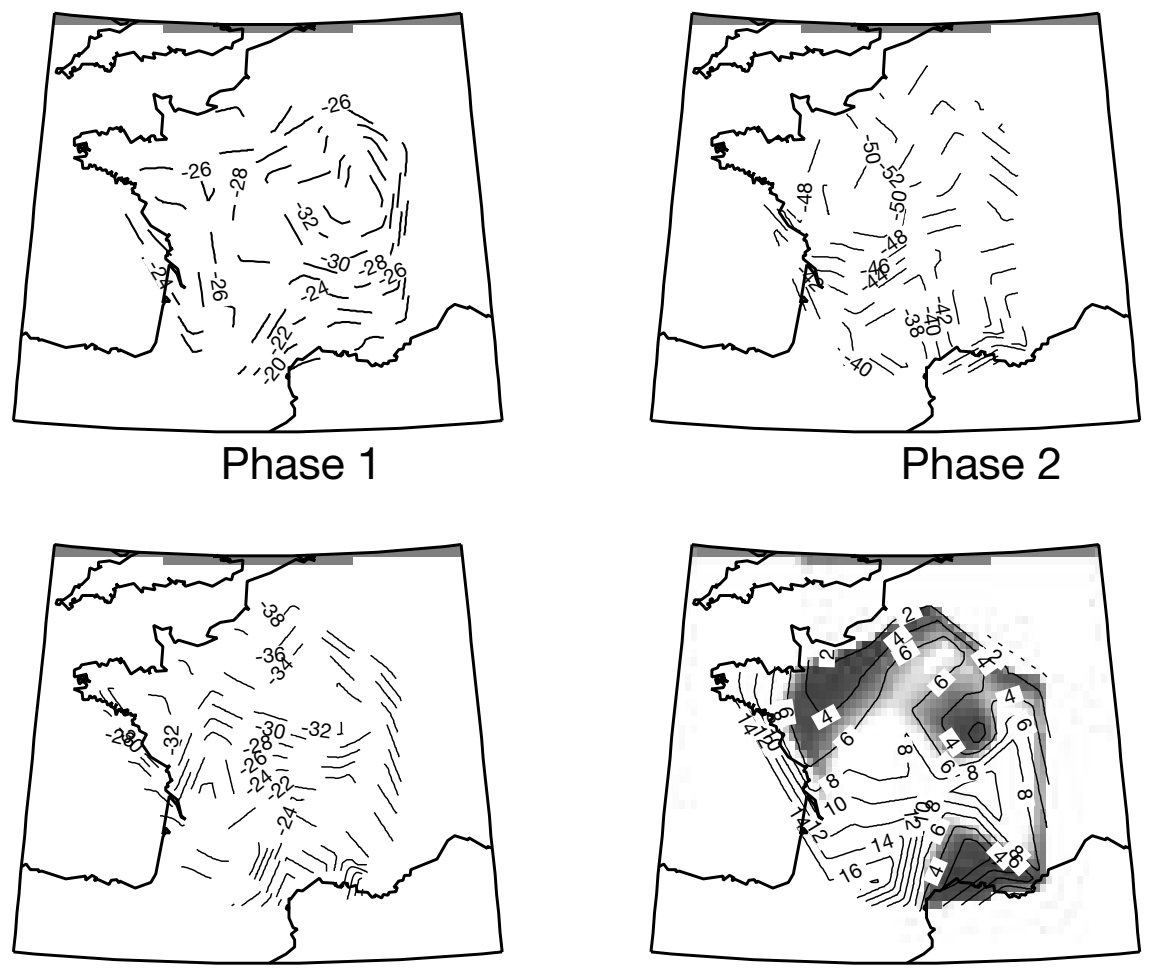

Phase 4
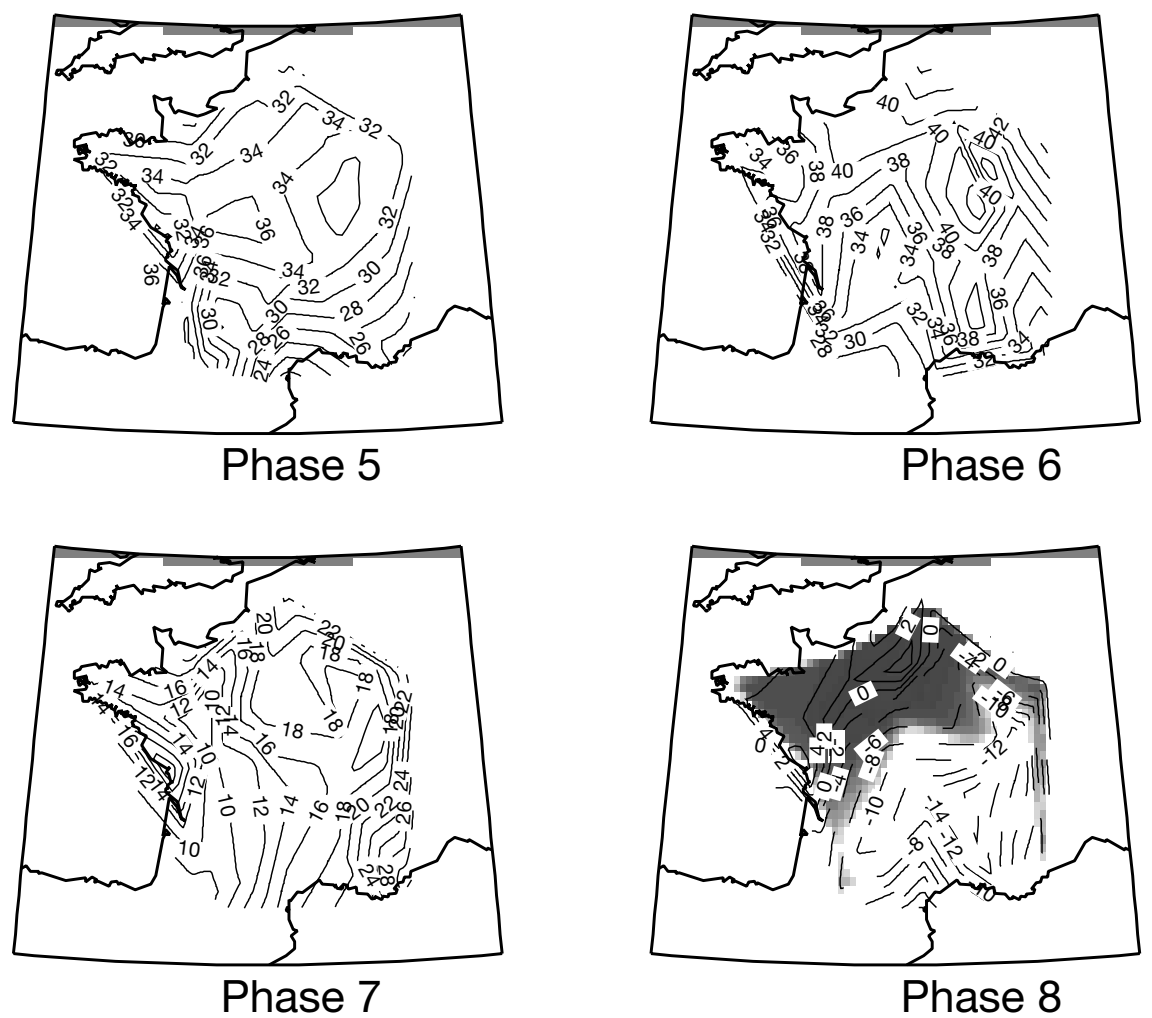

Fig. 4. Relation between the 8 phases of the $65 \mathrm{~d}$ oscillation and warm days (third tercile): contour levels (percentage deviation from the expected $33 \%$ ). The shaded area corresponds to regions where the deviations are between the 5 and $95 \%$ confidence levels 


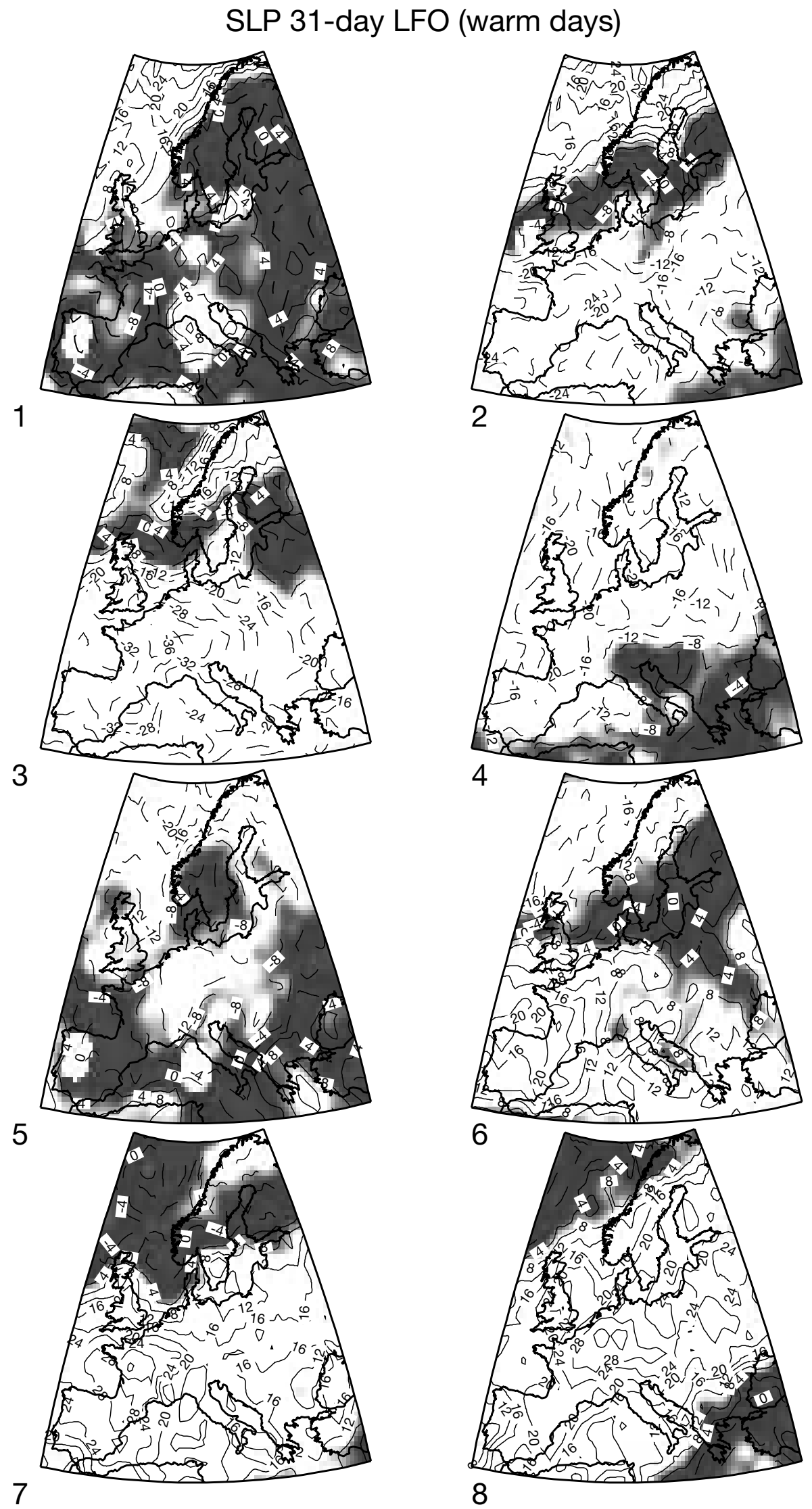

Fig. 5. Relation between the 8 phases of the $31 \mathrm{~d}$ oscillation and warm days, using the NCEP reanalysed data over the European sector (third tercile): contour levels (percentage deviation from the expected $33 \%$ ). The shaded area corresponds to regions where the deviations are between the 5 and $95 \%$ confidence levels 


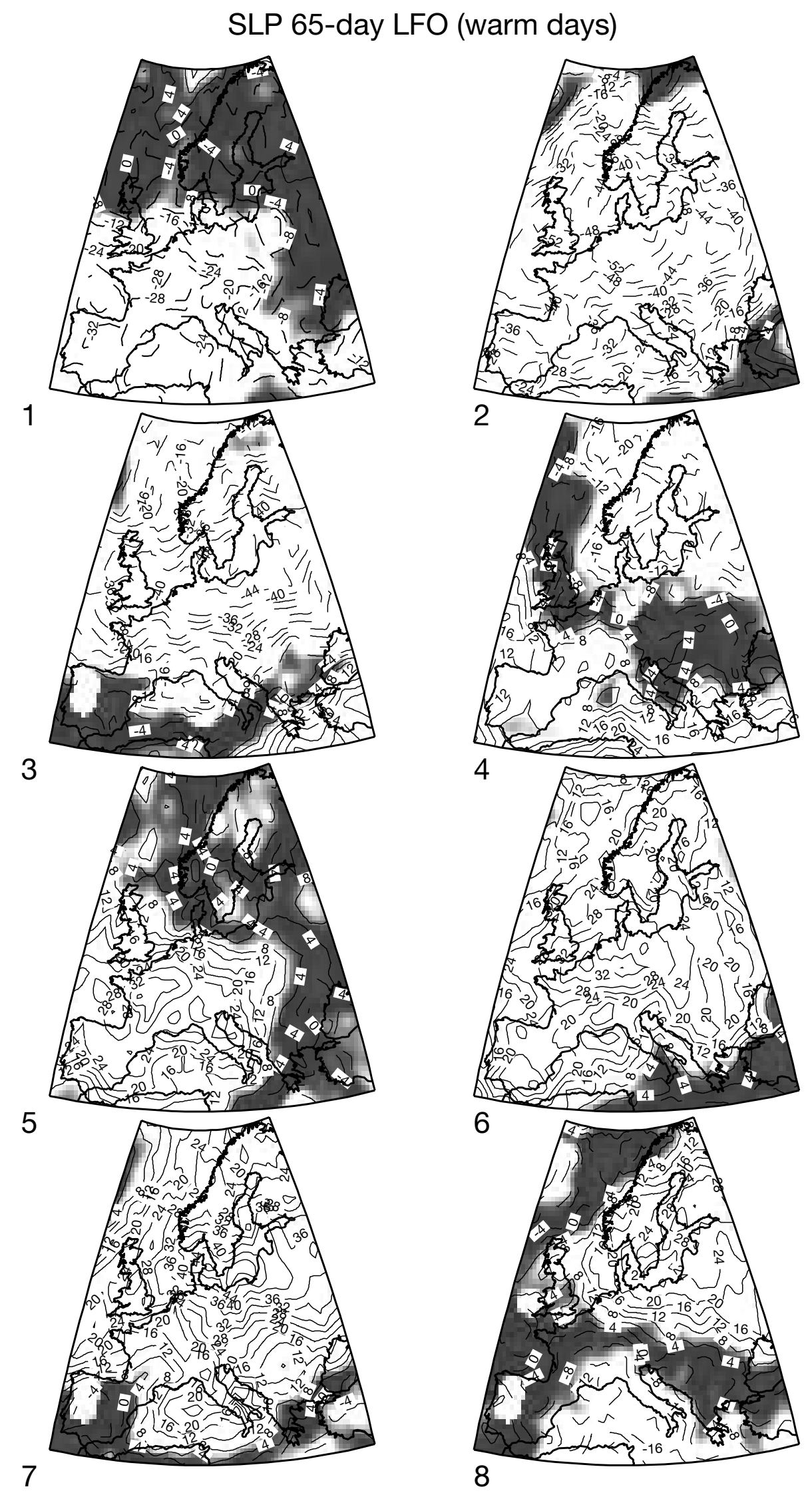

Fig. 6. Relation between the 8 phases of the $65 \mathrm{~d}$ oscillation and warm days, using the NCEP reanalysed data over the European sector (third tercile): contour levels (percentage deviation from the expected $33 \%$ ). The shaded area corresponds to regions where the deviations are between the 5 and $95 \%$ confidence levels 
ference which characterizes the $65 \mathrm{~d}$ oscillation, compared to the $31 \mathrm{~d}$ oscillation. The nonlinear interaction between the 2 modes is also visible in Fig. 4: phases 2, 3 and 5 have more impact on the local temperatures than the opposite phases 6,7 and 1 .

The same experiment was performed using NCEP reanalysed temperature data for the European aera and for the same period. The results are shown in Fig. 5 for the $31 \mathrm{~d}$ LFO and Fig. 6 for the $65 \mathrm{~d}$ LFO. The tendencies are very similar to the above results when restricted to France. Note that the influence of the $31 \mathrm{~d}$ mode over the very northern part of Europe is significantly reversed (phases 1, 2 and 3). A similar tendency is apparent for the $65 \mathrm{~d}$ mode in phases 4 and 8 between western and northeastern Europe. The $65 \mathrm{~d}$ mode interacts significantly more than the $31 \mathrm{~d}$ mode. This concordance of results between Météo-France station measurements and NCEP reanalysis was not $a$ priori obvious. It indeed confirms that NCEP reanalysis largely follows the observed climatology dynamics.

\subsection{Precipitation}

We now consider precipitation data collected from the 30 Météo France stations; we consider also 3 equally populated terciles of wet days (weak, normal and heavy events) together with a fourth category corresponding to dry days. We then use the same methodology as that used for the temperatures. The result for the $31 \mathrm{~d}$ mode is shown in Fig. 7 ; it represents deviations (in percentages) from the climatological mean for heavy precipitation (third tercile). Once again phases 2 and 3, linked to WBL regimes, contain significantly less (heavy) wet days than average. The Perpignan station exhibits the reversed tendency, due to the occurrence of a pronounced local climate in the Roussillon region, where easterlies often generate heavy or even extreme precipitation. Phases 6 and 7 contain more heavy wet days than average, except in the very south of France (Perpignan and Nice), where the tendency is reversed as for phases 2 and 3. The same difference is also observed in Plaut \& Simonnet (2001), where the authors compared local precipitation and Z700 LSC WRs. The $65 \mathrm{~d}$ oscillation has weaker interactions with the precipitation data, with less significant contour levels. The strongest interaction occurs for phase 2 (WBL regime), which gives results very similar to the $31 \mathrm{~d}$ mode (Fig. 8): the 2 modes' phases are in this case the same, leading to an enhanced positive anomaly in the west of Europe (the blocking pattern). We also observe the same reverse tendency for the $65 \mathrm{~d}$ oscillation, although extending more to the north than for the $31 \mathrm{~d}$ mode and including the Roussillon region.

\section{CONCLUSION}

The North-Atlantic, Western Europe sector low-frequency variability was studied by searching for specific space-time patterns in the flow. MSSA has enabled us to extract at least 3 robust oscillations on the intraseasonal time scale: a $31 \mathrm{~d}$ retropropagating mode as well as 65 and 120-130 d oscillations with northward propagating anomalies. The study of 3 different fields, namely SLP and geopotential heights (Z700 and Z500), indicates that these oscillations are almost similar and occur at the same time. Moreover, these 3 barotropic oscillations are most often phase-locked. The 31 and 65 $\mathrm{d}$ modes are indeed likely to be harmonics of the $130 \mathrm{~d}$ oscillation. A $42 \mathrm{~d}$ mode seems also to be present in geopotential quantities and to a lesser extent in SLP. No baroclinic modes emerge at this time scale.

Next, we studied the relationship between these oscillations and the succession of WRs defined in Plaut \& Simonnet (2001) as the most recurrent atmospheric states using the dynamical cluster algorithm (Michelangeli et al. 1995). The simultaneous occurrence of a given WR and 1 phase of either the 31 or $65 \mathrm{~d}$ modes indicates that the succession of WRs is largely influenced by the presence of these oscillations. We thus observe that the preferred transitions for the 5 SLP WRs are $\mathrm{ZO} \rightarrow \mathrm{BL}(\mathrm{AR}) \rightarrow \mathrm{WBL} \rightarrow \mathrm{GA} \rightarrow \mathrm{ZO}$. It is striking to observe that these 2 oscillations alone are able to explain most of the preferred and forbidden transitions, although they are quite intermittent. This is a confirmation of the results found independently in Plaut \& Vautard (1994) with the 'quasi-stationary' $Z 700$ regimes (Vautard 1990).

We were able to show that the occurrences of the different phases of the 31 and 65 d oscillations have a statistically significant influence over local climates (temperatures as well as precipitation). Local departures from the climatic averages are most often highly correlated to the life cycle of these intraseasonal oscillations. We thus obtain a result very similar to Plaut \& Simonnet (2001), but where the interactions of the occurrences of WRs with local climates is studied instead. This is not surprising in the sense that our LFOs can be well described in terms of these WRs.

The links between LFO and WR approaches are less simple than it appears. The concept of WRs holds some arbitrariness, in the sense that they have no unique definition; however, different definitions lead to very similar large-scale patterns (Michelangeli et al. 1995). In all ways, the WR provide a very convenient way to describe atmospheric states. On the other hand, LFOs can be searched for by a procedure that can be applied to any dynamical system. They also originate from specific physical phenomena. There is evidence that the annual frequencies of some of our WRs are directly in- 


\section{SLP 31-day LFO (wet days)}

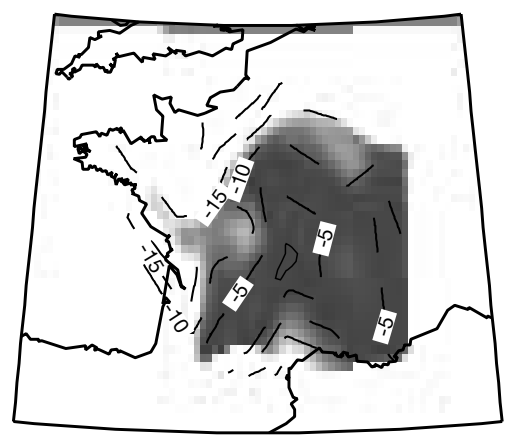

Phase 1

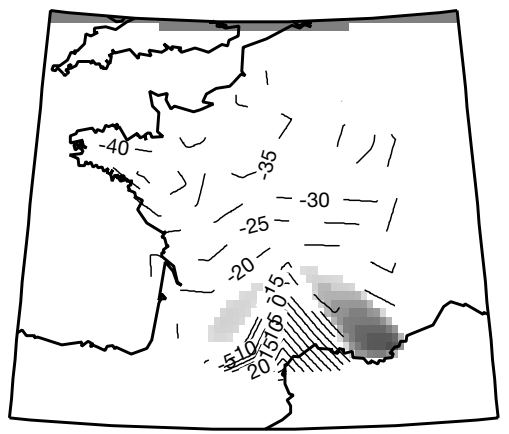

Phase 3

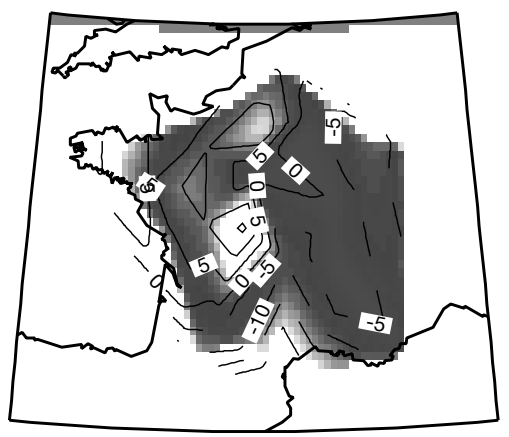

Phase 5

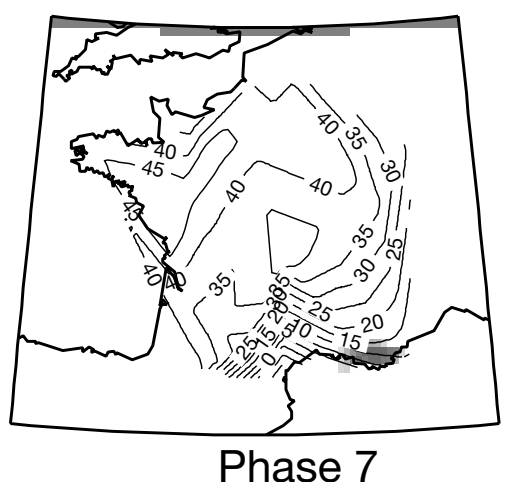

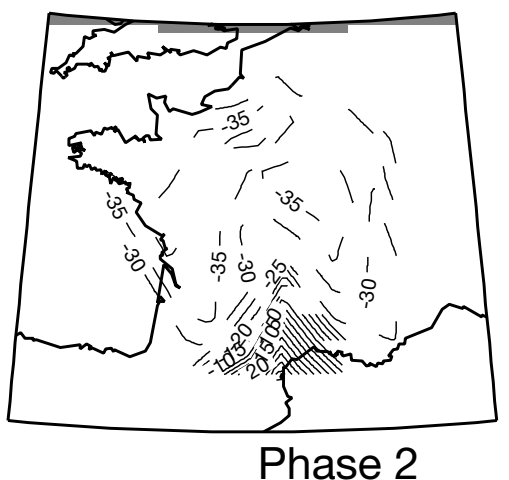
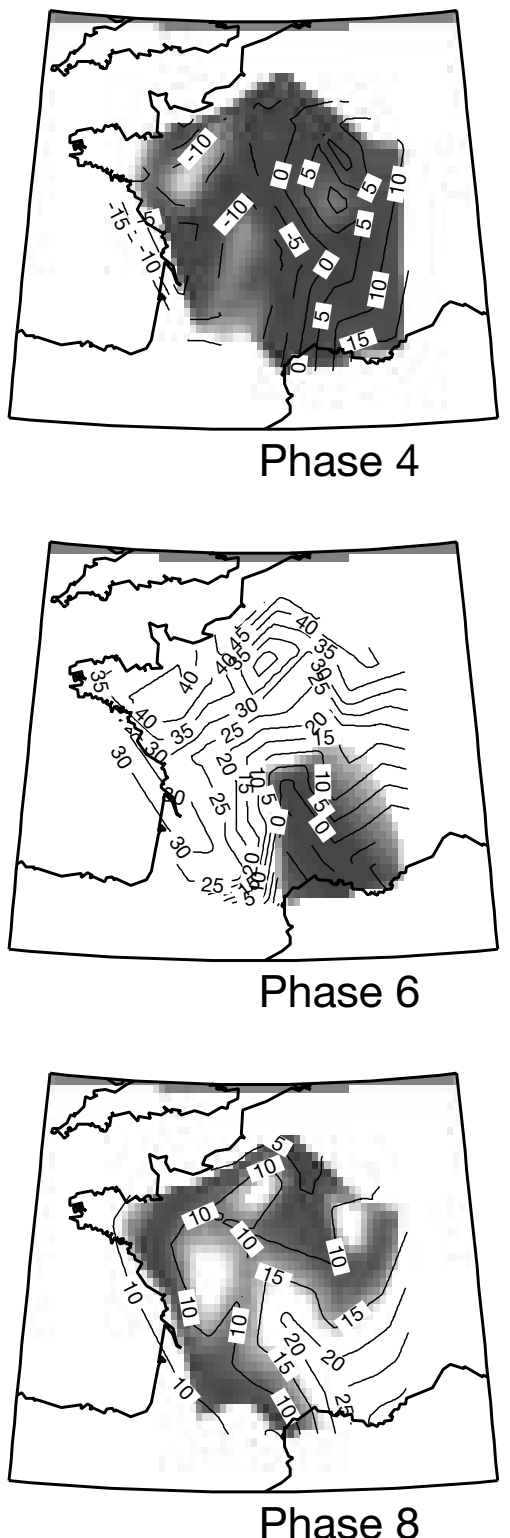

Fig. 7. Relation between the 8 phases of the $31 \mathrm{~d}$ oscillation and wet days (third tercile): contour levels (percentage deviation from the climatological mean). The shaded area corresponds to regions where the deviations are between the 5 and $95 \%$ confidence levels 


\section{SLP 65-day LFO (wet days)}

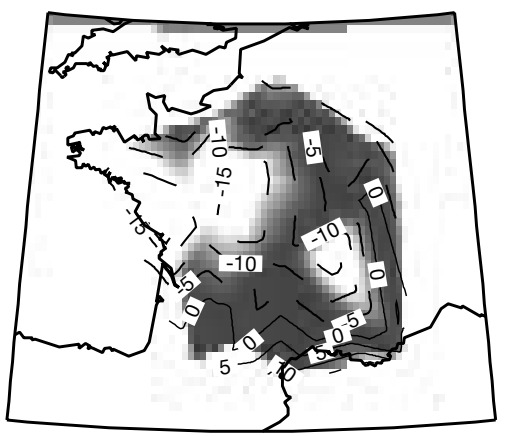

Phase 1

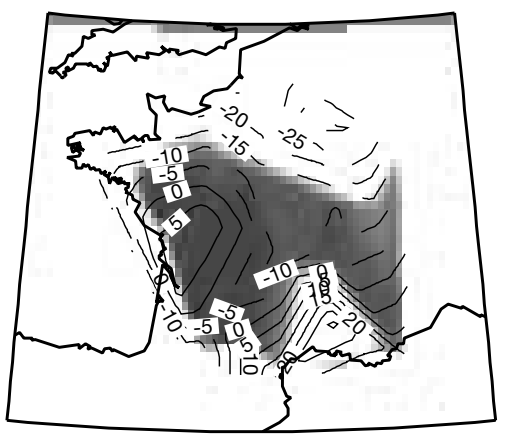

Phase 3

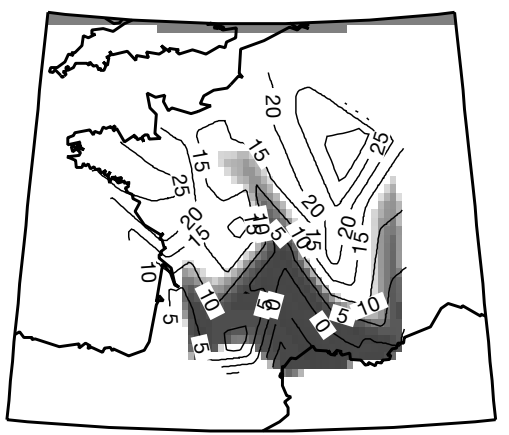

Phase 5

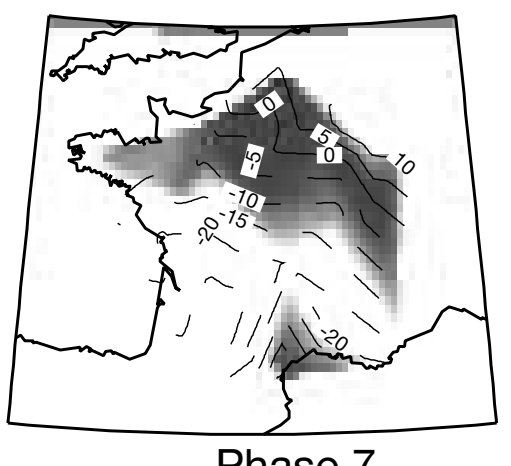

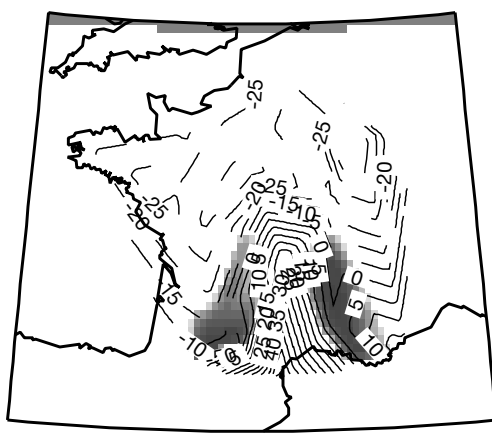

Phase 2

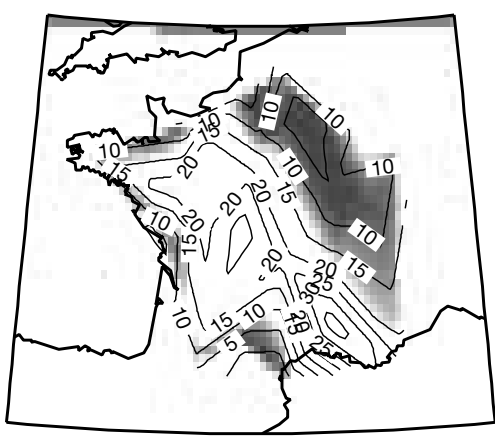

Phase 4

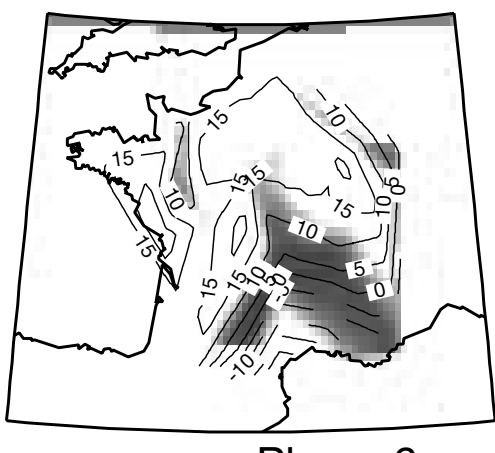

Phase 6

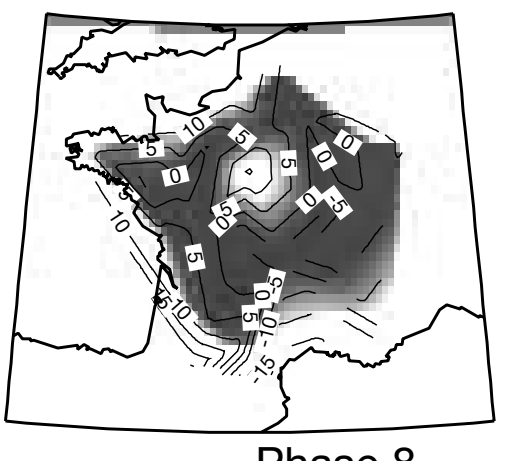

Phase 8

Fig. 8. Relation between the 8 phases of the $65 \mathrm{~d}$ oscillation and wet days (third tercile): contour levels (percentage deviation from the climatological mean). The shaded area corresponds to regions where the deviations are between the 5 and $95 \%$ confidence levels 
fluenced by interannual and even interdecadal SLP LFOs (Simonnet unpubl.), but these links somehow depend on how the WRs are defined. It appears that the intraseasonal oscillations we detected could also be influenced by a much lower frequency dynamics. In a preliminary study, a combination of wavelet transform and MSSA enabled us to detect fluctuations of the amplitudes of the previous oscillations in a time-frequency domain over the last $120 \mathrm{yr}$. A robust oscillation of 7-8 yr was thus detected in the modulation of LFOs amplitude, that is with the same period as the 7-8 yr northern hemisphere oscillation found in several independent studies (Plaut et al. 1995). However, direct influences of interannual oscillations over intraseasonal oscillations still need to be established, assuming they exist.

Acknowledgements. We would like to thank the CRU for making their 120 yr SLP daily data set NCEP reanalyses available to us. G.P. thanks Météo-France for the French station daily observations data set.

Submitted: March 6, 2000; Accepted: August 22, 2000

\section{LITERATURE CITED}

Allen MR, Smith LA (1996) Monte Carlo SSA: detecting irregular oscillations in the presence of colored noise. J Clim 9: 3373-3404

Jones PD (1987) The early twentieth century Arctic Highfact or fiction? Clim Dyn 1:63-75

Michelangeli PA, Vautard R, Legras B (1995) Weather regimes: recurrence and quasi stationarity. J Atmos Sci 52: $1237-1256$

Plaut G, Simonnet E (2001) Large-scale circulation classification, weather regimes, and local climate over France, the Alps, and Western Europe. In: von Storch H, Jones P (eds) ACCORD: Atmospheric circulation classification and regional downscaling. CR SPECIAL 9. Clim Res 17:303-324

Plaut G, Vautard R (1994) Spells of low-frequency oscillations and weather regimes in the Northern Hemisphere. J Atmos Sci 51:210-235

Plaut G, Ghil M, Vautard R (1995) Interannual and interdecadal variability in 335 years of Central England temperature. Science 268:710-713

Vautard R (1990) Multiple weather regimes over the North Atlantic. Analysis of precursors and successors. Mon Weather Rev 118:2056-2081

Proofs received from author(s): May 25, 2001 